\title{
GWAS, Cytomegalovirus Infection, and Schizophrenia
}

\author{
Jakob Grove • Anders D. Børglum • Brad D. Pearce
}

Published online: 25 September 2014

(C) Springer International Publishing AG 2002

\begin{abstract}
In recent years, good progress has been made in uncovering the genetic underpinnings of schizophrenia. Even so, as a polygenic disorder, schizophrenia has a complex etiology that is far from understood. Meanwhile, data are being collected enabling the study of interactions between genes and the environment. A confluence of data from genetic and environmental exposure studies point to the role of infections and immunity in the pathophysiology of schizophrenia. In a recent study by Børglum et al., a single nucleotide polymorphism (SNP) in the gene CTNNA3 was identified that may provide clues to gene-environment interactions. The carriers of the minor allele for the SNP had a fivefold risk of later developing schizophrenia if their mothers were CMV positive, while the children not carrying the allele had no excess risk from maternal CMV. In the current paper, we summarize recent advances to clarify a possible mechanism of such interactions between the host genotype and infection in schizophrenia risk.
\end{abstract}

J. Grove · A. D. Børglum

Department of Biomedicine, Aarhus University, Bartholins Allé 6,

Bldg. 1242, 8000 Aarhus C, Denmark

J. Grove

e-mail: grove@biomed.au.dk

A. D. Børglum

e-mail: anders@biomed.au.dk

J. Grove

Bioinformatics Research Centre, Aarhus University, Aarhus,

Denmark

J. Grove · A. D. Børglum

iPSYCH, The Lundbeck Foundation Initiative for Integrative

Psychiatric Research, Aarhus, Denmark

J. Grove · A. D. Børglum

iSEQ, Centre for Integrative Sequencing, Aarhus University, Aarhus, Denmark
Keywords Schizophrenia · Genome-wide association studies · Cytomegalovirus · Genetics · Gene-environment interactions $\cdot$ Immunogenetics $\cdot$ Autoimmunity $\cdot$ Antibody

\section{Introduction}

Schizophrenia (SCZ) is a serious debilitating, chronic brain disorder characterized by psychosis, disorganized thinking and behavior, and "negative" symptoms. It affects approximately $1 \%$ of the population worldwide and is one of the leading causes of years lost due to disability according to the WHO's Global Burden of Disease study. Pharmacological treatments do exist, but their efficacy is poor and they come with many adverse side effects [1].

Although the etiology of SCZ remains unknown, a congruent picture is beginning to emerge [2]. Recent studies suggest that environmental insults, superimposed on genetic

\section{A. D. Børglum}

Translational Neuropsychiatry Unit, Department of Clinical

Medicine, Aarhus University, Aarhus, Denmark

\author{
B. D. Pearce \\ Center for Translational Social Neuroscience, Emory University, \\ Atlanta, GA, USA
}


vulnerabilities, apparently act on the developing brain to trigger neurocircuitry abnormalities that ultimately lead to the neuropsychiatric symptoms of SCZ. In theory, the genome and environment could exert independent effects on $\mathrm{SCZ}$ pathogenesis. In some individuals, genetic variants or mutations may be responsible for the disorder, whereas in others, environmental factors may dominate. However, there is increasing evidence that genetic liabilities interact with environmental exposures [3].

Reports of a viral contribution to the etiology of SCZ go back as far as the early twentieth century when Karl A. Menninger published an article linking psychoses with schizophrenia-like symptoms to the 1918 influenza pandemic. But even before that it had been hypothesized that "almost every form of disease of the nervous system may follow influenza" [4]. Indeed, among environmental agents, microbial pathogens have gained prominence in the scientific literature based on epidemiological studies, animal models, and a growing recognition that immunogenetic factors are crucial in the pathophysiology of SCZ [5, 6]. The bulk of the research has focused on viruses and the intracellular parasite, Toxoplasma gondii $[7,8]$. However, this does not rule out a role for bacteria and other microbes, which could act in concert, or the role of a two-hit model in which an infection during neurodevelopment is followed later in life by an instigating infection that occurs near the time psychiatric symptoms become evident [6].

Pathogenic organisms are a ubiquitous and unavoidable environmental exposure for all humans. Moreover, infectionbased and genetic etiologies for SCZ are conceptually complementary because host genes determine individual susceptibilities and immune responses to viral infections, and thus genetic variables likely help determine the neuropsychiatric sequela of a viral exposure [6].

\section{Genetics of Schizophrenia}

There is substantial evidence that genetic factors contribute to risk for SCZ, but the underlying genetic architecture and pathophysiology have not been discerned. Early genomewide association studies (GWAS) in SCZ were disappointing and found only few genome-wide significant loci, but it is now clear these studies were underpowered [9]. Nevertheless, there have been a few single nucleotide polymorphisms (SNPs) consistently associated with SCZ. As sample sizes progressively increased, so did the number of SNPs found genome-wide significantly associated with $\mathrm{SCZ}$. In the most recent SCZ GWAS from the Psychiatric Genomics Consortium (PGC) encompassing 36,989 cases and 113,075 controls, 108 loci were identified, 83 of which had not previously been linked to SCZ [10••]. This leaves little doubt that SCZ is a complex polygenic disorder. Moreover, the combined sample size has now passed a threshold where the number of associated loci as a function of sample size grows at a rate similar to that of complex somatic traits such as height and Crohn's disease.

Recently, it has been in vogue to attempt to encode the combined effect of common SNPs into a risk profile score (RPS). An RPS is defined by estimating the SNP effects in a training data set and applying these effects as weights in a weighted sum of trait-associated alleles for each individual in the target data set.

Purcell et al. [11•], who were the first to show the utility of RPS in SCZ, found strong association with SCZ. Moreover, their results indicated that a large number of truly associated loci lie within the body of non-significant markers encouraging further collaborative GWAS in SCZ. Indeed, this has been confirmed in subsequent studies with increasing sample sizes $[12,13,10 \bullet \cdot$. As sample sizes grow, RPS will provide an estimate of heritability that will converge towards the true heritability due to common variants [14]. It is, however, still poor at predicting individual risk, as demonstrated in a Danish sample [10••]. Another area where RPS has been employed is in cross-disorder studies. Here it has demonstrated the substantial genomic overlap with other psychiatric disorders [15].

Effect sizes observed for common SNPs in SCZ are generally small with odds ratios typically about $1.1-1.2$.This is probably not surprising with the SNP markers tagging common variation. If there were common variants with higher effect sizes, they presumably would have been detected in the earlier, smaller GWAS. On the other hand, rare variants require a large $\mathrm{OR}$ to be detected. Indeed, the rare copy number variations (CNVs) found in GWAS generally have a much stronger association with high odds ratios, for example, 16 for deletion $15 \mathrm{q} 13.3$ [16] and more than 20 for deletion $22 \mathrm{q} 11.2$, the strongest known genetic risk factor for SCZ [17-19].

Next generation sequencing is a promising technology to identify rare, single nucleotide variations as well as structural variants, but such studies to date have been underpowered for genome wide searches of association. The studies that have been successful in identifying rare variants associated with SCZ have either been focused at candidate regions based on current literature, such as [20], or they have concentrated on de novo events [21-24], where de novo mutations have first been identified in families and then the frequency compared between SCZ patients and their healthy controls. No doubt future, large scale sequencing studies are going to identify more rare variants associated with SCZ.

\section{Human Cytomegalovirus}

As expected of a herpes virus, cytomegalovirus (CMV) is not cleared by the immune system, but instead causes a lifelong persistent infection that includes cycles of reactivation, which 
break the tenuous peace between the virus and its host [25]. Because CMV is spread through body fluids, including saliva, tears, vaginal fluids, semen, and breast milk, the infection is highly prevalent worldwide. In the United States and Europe approximately $50-85 \%$ of adults are infected [26].

Most cases of CMV are asymptomatic or cause only minor symptoms such as fever and self-limiting lymphandonopathy. Nevertheless, CMV is able to persist in diverse cell types including neural tissues [27]. Shedding of CMV is highest soon after primary infection but can continue for years, or may occur intermittently because of reactivation or reinfection with another strain of CMV [28].

In contrast to the benign course of CMV infection among immunocompetent children and adults, congenital infection is associated with substantial morbidity. CMV infection is the most common congenital infection, affecting $0.5-3 \%$ of all newborns $[29,30]$. Following primary maternal CMV infection, there is a 40 to $50 \%$ chance that the fetus will become infected [29]. This risk is substantially reduced if the mother has a latent or reactivated form of CMV infection, and the feto-protective role of maternal antibodies is of particular interest in the connection between CMV and SCZ (see below). The brain is a target of congenital CMV, but the ability of CMV to produce encephalitis with distinct neurological signs does not exclude this virus as the culprit in subtler neurobehavioral syndromes. For example, Houenou et al. recently found that higher CMV titers were associated with smaller hippocampal volume in patients with SCZ or bipolar disorder [31].

The humoral immune response to a primary CMV infection elicits the typical IgM response, which declines over several months as IgG titers rise. Hence, in the standard paradigm, IgM represents evidence of a recent infection, whereas IgG indicates infection had occurred any time in the past. However, this standard paradigm has been questioned in that reinfection or reactivation of an existing infection can (in some individuals) produce an IgM response [32]. Moreover, a high IgG titer does not necessarily connote strong immune control of CMV, but instead may represent a recent reactivation of the infection [25]. Thus, elucidating the relevant interaction of genetic polymorphisms with CMV exposure in SCZ etiology will rest on the interpretation of antibody levels. These antibodies themselves can be pathogenic, for example, via molecular mimicry between viral proteins and neural proteins in the host brain resulting in an autoimmune phenomenon, which has often been described in SCZ [33-35, 6].

\section{Environmental Risk Factors and Gene-Environment Interaction}

It is widely recognized that both genetic and environmental factors contribute to the etiology of SCZ. The essential role of environmental factors in the etiopathogenesis of SCZ has been demonstrated unequivocally in studies of concordance rates in monozygotic twins. Most well-controlled studies have yielded concordance rates of less than $50 \%$ (compared to a theoretical value of $100 \%$ if the $\mathrm{SCZ}$ was purely genetic) [36, 37].

As for gene-environment interactions (GxE), it is broadly acknowledged to play a role in complex disorders such as SCZ. Yet to our knowledge, the literature has only one genome wide study of GxE in SCZ with robust exposure information, namely [38••] (see below). The two main reasons are that GxE analysis demands larger samples than analysis of association, and more crucially, there are few large datasets where both environmental measurements and genome wide genotypic information are collected. Even candidate gene studies of GxE for SCZ and CMV seem quite limited [39] (see below). A separate avenue for research into GxE is animal models, which can be used to obtain experimental rather than observational evidence for GxE, see [40]. Animal models using mouse CMV have made extensive use of genetically manipulated mouse strains and have tested neuro-invasiveness, neurochemical changes, and behavioral sequela [41-43], but these have yet to be optimized to study SCZ susceptibility genes identified in recent GWAS studies. One avenue to optimizing this inquiry is to prioritize relevant pathways (see below), and work across disciplines. For example, a CNV affecting MAPK3 is associated with SCZ [44, 45], and in an unrelated animal model, this kinase was linked to CMV pathogenesis [46].

Epigenetics represents a possible conduit linking DNA sequence variation and environmental exposures to $\mathrm{SCZ}$ risk. Undoubtedly, a major mechanism by which diverse environmental exposures cause harm is by altering gene transcription. Epigenetics is a broad term that refers to mechanisms by which gene expression activity is regulated across meiosis and mitosis in the absence of underlying changes to DNA sequence.

While the role of epigenetics in immune regulation has been studied extensively, the potential relevance of this to SCZ is just beginning to be explored [47, 48]. Humans are constantly confronted with pathogens that mutate to avoid immune surveillance, and the host must adapt dynamically to this constantly changing threat. The diversity of MHC genes, and the exquisite machinery for antigen presentation, is one excellent example of this. But on a shorter timescale, epigenetic modulation of transcription in immune cells or other host tissues could also play a role.

The importance of immunogenetics in SCZ has come to the forefront now because the most consistent finding in large scale GWAS of SCZ has implicated immune genes, particularly - though not exclusively - genes in the MHC region. This has also been the case for the latest PGC study [10 • ] that showed enrichment for genes related to immunity as well as genes expressed in the brain. 
The rationale for a causal role of immunological factors in SCZ rests on a solid foundation showing that immune cells and immunomodulatory cytokines profoundly influence complex behaviors in humans and experimental animals, and immune cells and molecules interact with the developing CNS to cause latent perturbation of brain function and behavior $[6,49,50]$. Thus, there is a confluence of evidence from epidemiology, neuropathology, and genetics, which continues to strengthen the connection between viral infections, immune responses, and SCZ.

\section{GWAS of Interaction Between Maternal CMV and Offspring's Genotype}

In a Danish GWAS of SCZ, a single SNP was identified having an experiment-wide significant interaction with maternal CMV infection [38••]. Children carrying the minor allele of SNP rs7902091 had a fivefold increased risk of developing SCZ later in life if their mothers were CMV positive, while non-carriers had no excess risk.

The SCZ GWAS was based on diagnoses from the Danish Psychiatric Central Registry and conducted on dried blood spot samples on filter paper from the Danish Newborn Screening Biobank [51]. The analysis was carried out on a small subsample of the GWAS consisting of 488 cases and equally many controls where serological measurements of IgG antibodies to CMV had been conducted. As the blood samples were drawn for the most part within the first week after birth when the immune system of the child is not yet fully developed, the high level of IgG antibodies in a sample is taken as an indication of high levels of IgG antibodies in the mother.

The statistical analysis performed was the two-step method of Murcray et al.[52] who identified a single SNP, rs7902091, with a genome-wide adjusted $p$-value of 0.021 and an interaction odds ratio of 5.3 [95 \%CI $(2.75-10.4)]$. The locus harbored other SNPs relatively independent of rs7902091 that also showed signs of interaction although they did not meet global significance. There is the possibility that rs7902091 could be a false positive and replication in an independent, preferably larger, sample is warranted.

The SNP is located in an intron of the very large gene, CTNNA3, just upstream from the nested gene LRRTM3. CTNNA3 encodes catenin alpha-3, which mediates cell-cell adhesion linking cadherin-based cell-cell adhesion complexes and the cytoskeleton $[53,54]$. Smith et al. recently reviewed the unusual features of this genomic locus, as well as the complex transcriptional regulation affecting both CTNNA3 and the nested LRRTM3 gene [53]. In addition to being one of the largest genes in the human genome, CTNNA3 is regulated by multiple promoters and epigenetic gene silencing mechanisms. In CTNNA3 the leading SNP from the PGC GWAS was rs 186235844 , but as LD around rs7902091 decays rapidly and there are several recombination hotspots between them, the two SNPs are not in LD [10••].

In theory, SCZ-associated genetic loci could interact with $\mathrm{CMV}$ at any point in the causal chain of SCZ, from early neurodevelopment to the period of transition from prodromal phase to clinical illness, or even as an effect modifier of the symptom profile once the psychotic illness was established [6]. However, given the findings of Børglum et al., which suggest that maternal CMV infection becomes a risk factor for SCZ based on the genotype the progeny, our discussion is focused on maternal infections and the risk for the offspring.

There is a multitude of mechanisms by which CTNNA3 variants could interact with CMV to increase risk of SCZ, and further complicating this connection, the CMV antibody detected could be a marker for another infection that has a similar epidemiological pattern. Importantly, the exposure variable in this study was anti-CMV IgG, and we have no evidence that the fetus was actually infected. Moreover, the antibody repertoire to CMV can vary with time and between individuals in both neutralizing capacity and affinity. Studies are emerging that examine the maternal fetal interface and the role of different types of anti-CMV antibodies and their relationship to cadherin adhesion [55]. Polymorphisms in CTNNA3 could help determine the nuanced interactions between antibody and CMV infection in the placenta, and hence modulate the risk for neurodevelopmental adversity in the fetus.

\section{Integrating Finding from GWAS x CMV in the Broader Literature}

Despite the progress offered by recent GWAS studies of SCZ, it has remained difficult to ascertain which genetic variants are causative, and even more challenging to understand how their contribution meshes with environmental antecedents. Understanding this connection has been a prominent gap that has hindered innovations in both the prevention and treatment of SCZ. The clues provided by Børglum et al. point to a specific role for CTNNA3. This study correlates maternal anti-CMV $\mathrm{IgG}$ to the later development of SCZ in the offspring. Since the CTNNA3 risk alleles were genotyped in the offspring and not the mothers, a variety of mechanisms can be considered, and these can be better prioritized by considering the gene expression patterns of CTNNA3 (Fig. 1).

CTNNA3 is robustly expressed in the placenta and certain fetal tissues, including the brain (Fig. 1A). The placenta consists of tissues of both maternal and fetal origin. Although the interaction of CMV with CTNNA3 is based on genotyping of the child, the mother shares $50 \%$ of the child's genome, and hence it is possible that pathophysiological mechanisms involving CTNNA3 variants could involve the mother carrying the minor allele, and the child's genotype may merely be an indication of these shared SNPs. Hence, parent of origin studies would be 
Fig. 1 Results of expression levels for CTNNA3 in various human tissues. The figure shows normalized gene expression levels from the NextBio Body Atlas database. The score at the top of each panel is obtained as described: see http://www. nextbio.com/b/nextbioCorp.nb. First, a per-chip median normalization on probe sets common to all platforms was performed and then combined using quantile normalization. Intensities for probe sets unique to particular platforms were rescaled to the same per-chip median, fit by linear interpolation, using the intensities of the common probe sets between platforms as a reference. Median score across all tissues was 584. Panel A: fetal and progenitor cells and tissues. Panel B: select tissues in adult
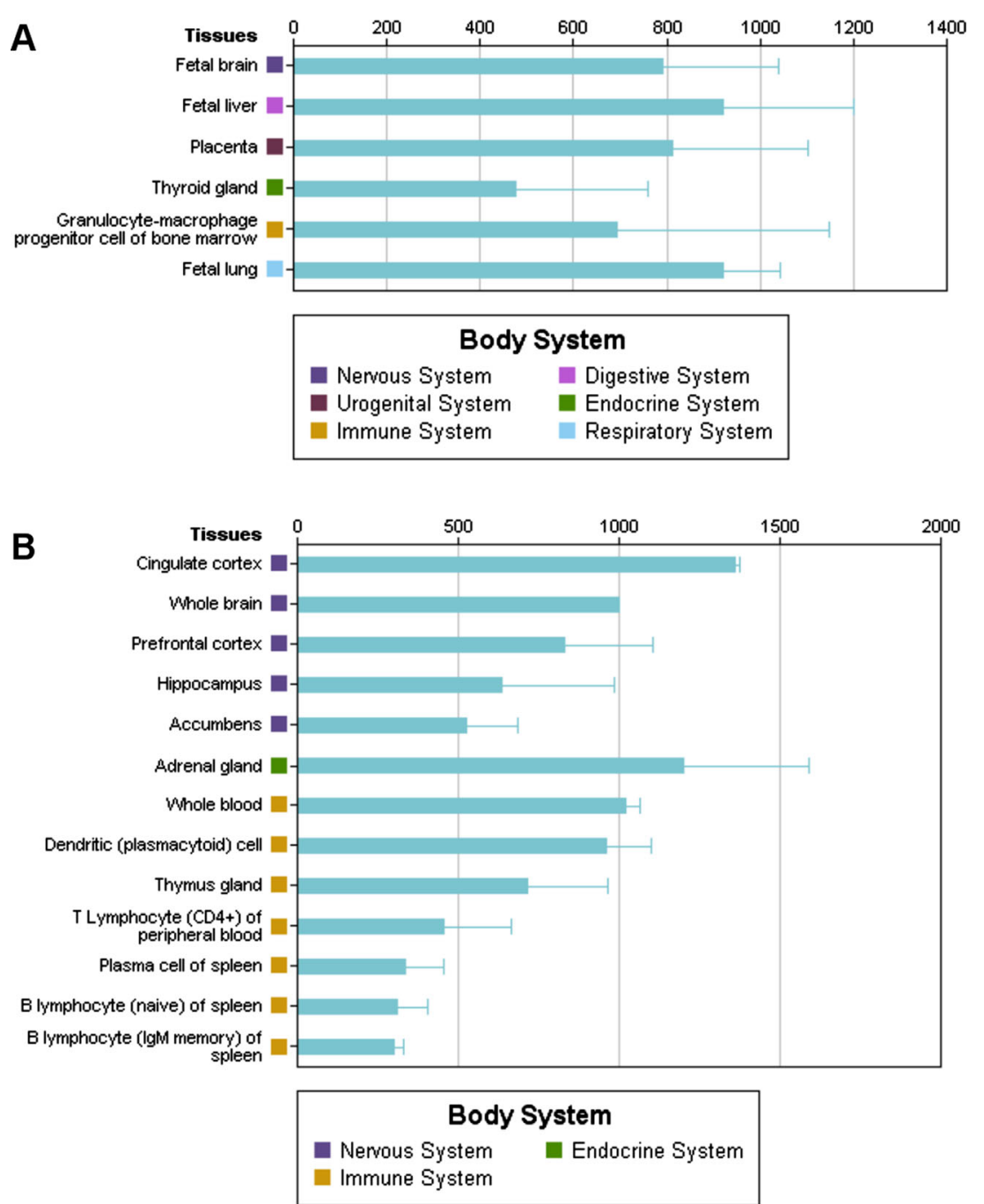

needed to sort this out, especially in light of the gene imprinting mechanism described below. In any case, CTNNA3 plays a crucial role in placental development $[56,54]$. The cadherincatenin complex is involved in orchestrating trophoblast invasion as well as assuring the integrity of the placenta across stages of gestation. Transcriptional regulation of CTNNA3 in the placenta is exquisitely regulated at the level of cell-type, and changes dynamically with gestational age [54].There is growing interest in the epigenetic regulation of this gene in the placenta, and its role in pregnancy complications such as preeclampsia $[57,54]$. Cytomegalovirus infection may be one such pregnancy complication that dysregulates CTNNA3 expression.

Genomic imprinting can be considered an extreme form of epigenetic regulation in that either the paternal or maternal allele is selectively silenced. The placenta is exceptional in both the number and pattern of imprinted genes [57]. CTNN A3 has complex genetic organization and is known to be imprinted in the placenta [54]. This places the regulation of its expression (e.g. through epigenetic mechanisms) in a crucial mechanistic position for connecting CMV to placental pathology, and perhaps ultimately to SCZ. Elucidation of relevant methylation quantitative trait loci (for CTNNA3 and its regulators) will further this line of inquiry as more is understood concerning the impact of CMV on host genes.

Mechanistically, CMV infects cytotrophoblasts and disrupts cell adhesion causing abnormalities in their invasion, and ultimately undermines placental integrity [55]. Thus, if the risk alleles found by Børglum at al. resulted in abnormal expression of CTNNA3 (or one of its isoforms), it could render the placenta less resilient to the CMV infection. One mechanism by which CMV has a deleterious impact on the fetus is through placental hypoxia, and hypoxia due to diverse maternal conditions has been connected epidemiologically to SCZ [58].

Conversely, CTNNA3 is also expressed in the fetal brain (Fig. 1A), and inhibits wnt signaling [59, 60]. CMV also regulates wnt signaling pathways [61]. Given the critical role of this signaling pathway in neurodevelopment, polymorphisms in CTNNA3 could have a deleterious effect on the compensation or resilience of the brain in fetuses exposed to CMV. Conversely, the immature fetal blood-brain barrier may 
be influenced by CTNNA3 expression, and this could allow pernicious anti-CMV antibodies from the mother to cross into the fetal brain and disrupt neurodevelopment without actual infection of the fetus [62]. Along these lines, there is accumulating evidence supporting a role of CTNNA3 in autism spectrum disorders (ASD).

As recently reviewed by Bacchelli et al., CTNNA3 is a candidate gene for ASD based on studies of SNPs, CNVs, and a rare compound heterozygous exon deletion [59]. Moreover, the alpha-catenin protein is expressed in a developmentally regulated pattern in the hippocampus and cerebral cortex [59]. This suggests a role for CTNNA3-mediated cell adhesion in the developing brain, and is in concert with diverse data sources showing the importance synaptic adhesion mechanisms in ASD [63].

The adult brain also expresses CTNNA3 (Fig. 1B). Thus polymorphisms in this gene could affect the resilience or compensatory capacity of neural circuitry that was disrupted in utero during gestation by CMV (or associated antibodies). This could play out in various postnatal periods as the malleable neurodevelopmental trajectory of a person at high risk for SCZ becomes manifested. Oxidative stress, or infection of the adult proband with another virus, could initiate a pathophysiological cascade leading to progressive instability of neurocircuitry that had previously been rendered vulnerable by the maternal CMV infection [64]. The possible role of CTNNA3 in neurocircuitry abnormalities of the adult brain is implied by the putative association of this locus with amyloid levels and late onset dementia $[65,66]$.
Several immune cells also express CTNNA3 (Fig. 1B), and hence this molecule is well poised to mediate neuroimmune interactions that could link CMV infection to the ultimate development of SCZ. Since the environmental marker in this study is the CMV antibody, it is possible that the GxE effect observed is mediated by B-cells or related pathways that influence the production, isotype, placental passage, bloodbrain permeability, or antigen affinity of antibodies. This hypothesis is supported by a recent GWAS study that found a CTNNA3 SNP (rs1786929) was associated with autoantibody production in occupational asthma [67].

An important consideration is also the nested gene, LRRTM3, which is strongly expressed in the brain and has been suggested to play a role in synaptic development and neural plasticity [53]. Accordingly, polymorphisms in this gene, which is proposed to have cell adhesion properties like CTNNA3, could result in an ineffective neuro-compensatory response to CMV infection.

\section{Data Integration for GxE}

The polygenic burden of SCZ implied by the largest and most recent GWAS [10••] suggests that these genes operate in complex molecular pathways. One or more of these pathways may involve CTNNA3. To explore possible pathways we uploaded the 362 genes highlighted by Ripke et al. in their SCZ GWAS [10••] into Ingenuity systems software (IPA), and examined their predicted connections with CTNNA3 in a

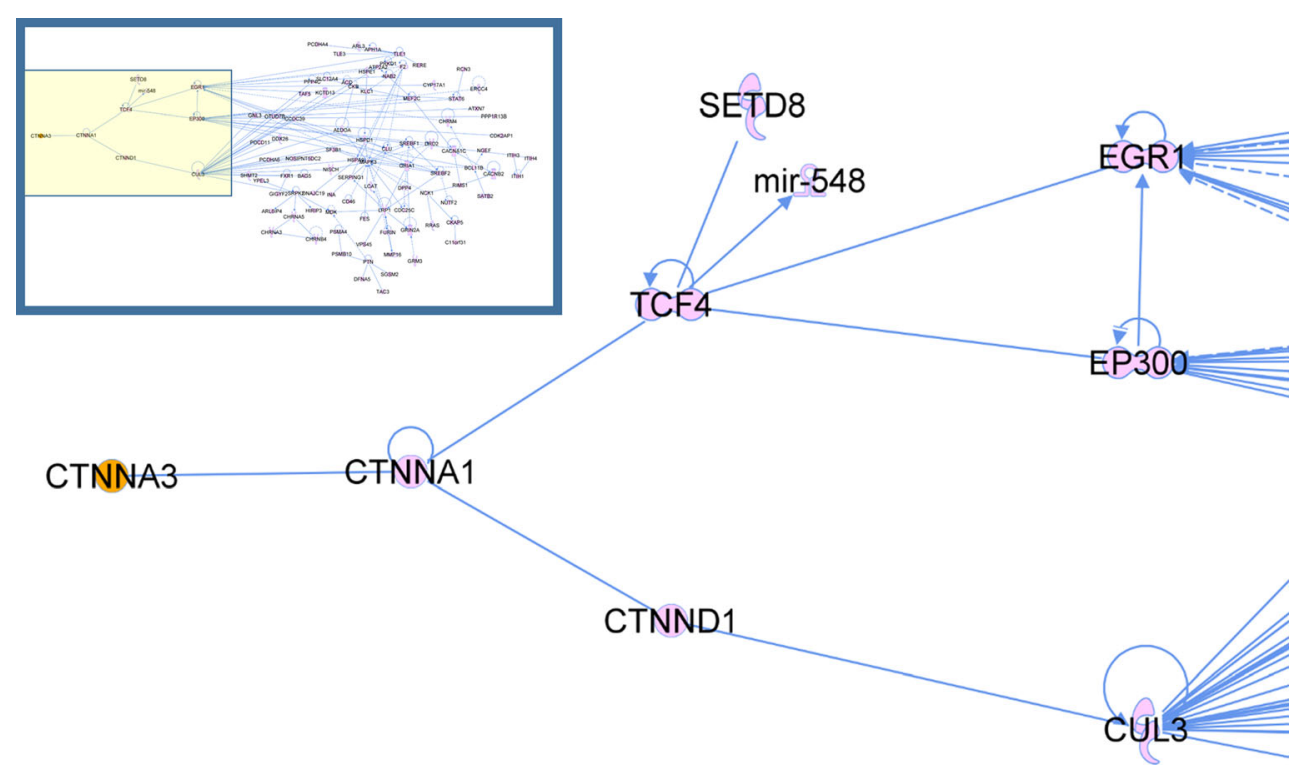

Fig. 2 Diagram showing predicted molecular connections between CTNNA3 and genes identified in a recent PGC GWAS of SCZ (doi:10. 1038/nature13595). The inset diagram shows the predicted interactome for the uploaded gene set. The magnified portion of the diagram is delimited by the yellow box and is shown as a detailed node-edge diagram, where arrows indicate the direction of the interaction, and lines without arrowheads indicate direct interaction in which directionality cannot be inferred (e.g., binding). Divergent lines extending to the right depict further connections with other SCZ genes in the PGC GWAS, which form an extensive network as shown in the inset panel. See http://www.ingenuity.com/ for information regarding the IPA database and algorithms 
node-link diagram using the IPA connect function (Fig. 2). As shown, CTNNA3 was predicted to be linked to other SCZ susceptibility genes through CTNNA1, which was a binding partner for TCF4, and CTNND1. These in turn were linked to numerous other candidate genes in the set via EGR1, EP300, and CUL3. While these data are based on the curated interactions in the IPA database, they probably do not take place in all tissues. Hence, this analysis is just a starting point for further laboratory study involving this hypothesized pathway.

\section{Future Outlook}

While genome-wide studies of GxE have been conducted, they are still generally rare and underpowered. High throughput genotyping is widely used, but there is general shortage of data sets including both genotypes and environmental variables. The technologies to subsequently tease out the biological mechanisms are also present even if there may be a demand for new high throughput methods. In the meantime, creative use of available samples holds the potential to test pathophysiological mechanisms.

The development in genetics over the past few years, where big consortia such as the PGC have pooled together a massive data set, has been driving the progress towards more and more robust GWAS findings and more and more loci. There is a need for a similar collaborative effort when it comes to environmental markers. Many high throughput methods are already available, but more will have to be developed. Moreover, universal standards of measurements and data structures are a necessity in order to enable the sharing and pooling of data between research groups and between countries. Unlike the genetic makeup, environments change over time. A particular challenge for future GxE studies of SCZ is, therefore, to provide measurements longitudinally on a large scale. Stored DNA can be used for epigenetic (DNA methylation) studies, though this may be highly dependent on which candidate gene is examined. The growing interest to allelespecific DNA methylation implies another productive avenue to test GxE interactions.

Acknowledgements This study was funded by the Lundbeck Foundation and by Aarhus University, both in Denmark. We are grateful for support from National Institutes of Health (NIMH) grant 1R21MH083138.

\section{Compliance with Ethics Guidelines}

Conflict of Interest Anders D Børglum and Jakob Grove have no conflicts of interest. Brad Pearce received a grant from the NIH.

Human and Animal Rights and Informed Consent This article does not contain any studies with human or animal subjects performed by the authors.

\section{References}

Papers of particular interest, published recently, have been highlighted as:

- Of importance

•- Of major importance

1. Lieberman JA, Stroup TS, McEvoy JP, Swartz MS, Rosenheck RA, Perkins DO, et al. Effectiveness of antipsychotic drugs in patients with chronic schizophrenia. N Engl J Med. 2005;353(12):1209-23. doi:10.1056/NEJMoa051688.

2. McGrath JJ, Mortensen PB, Visscher PM, Wray NR. Where GWAS and epidemiology meet: opportunities for the simultaneous study of genetic and environmental risk factors in schizophrenia. Schizophrenia bulletin. 2013;p. sbt108.

3. Uher R. Gene-environment interactions in common mental disorders: an update and strategy for a genome-wide search. Soc Psychiatry Psychiatr Epidemiol. 2014;49(1):3-14. doi:10.1007/ s00127-013-0801-0.

4. Menninger KA. Psychoses associated with influenza: i. general data: statistical analysis. J Am Med Assoc. 1919;72(4):235-41.

5. Brown AS. Epidemiologic studies of exposure to prenatal infection and risk of schizophrenia and autism. Dev Neurobiol. 2012;72(10): 1272-6.

6. Pearce BD. Can a virus cause schizophrenia: Facts and hypotheses. Neurobiological Foundations of Aberrent Behaviors. Boston: Kluwer Academic Publishers; 2003.

7. Mednick SA, Machon RA, Huttunen MO, Bonett D. Adult schizophrenia following prenatal exposure to an influenza epidemic. Arch Gen Psychiatry. 1988;45(2):189-92.

8. Yolken RH, Torrey EF. Are some cases of psychosis caused by microbial agents? A review of the evidence. Mol Psychiatry. 2008;13(5):470-9.

9. Kendler K. A joint history of the nature of genetic variation and the nature of schizophrenia. Molecular Psychiatry. 2014;

$10 . \bullet$ Schizophrenia Working Group of the Psychiatric Genomics Consortium. Biological insights from 108 schizophreniaassociated genetic loci. Nature. 2014;511(7510):421-7. doi:10. 1038/nature 13595.

11. Purcell SM, Wray NR, Stone JL, Visscher PM, O’Donovan MC, Sullivan PF, et al. Common polygenic variation contributes to risk of schizophrenia and bipolar disorder. Nature. 2009;460(7256): 748-52. doi:10.1038/nature08185.

12. Ripke S, Sanders AR, Kendler KS, Levinson DF, Sklar P, Holmans $\mathrm{PA}$, et al. Genome-wide association study identifies five new schizophrenia loci. Nat Genet. 2011;43(10):969-76. Available from: http://www.nature.com/ng/journal/v43/n10/full/ng.940.html.

13. Ripke S, O’Dushlaine C, Chambert K, Moran JL, Kahler AK, Akterin S, et al. Genome-wide association analysis identifies 13 new risk loci for schizophrenia. Nat Genet. 2013 aug;p. -. Available from: http://dx.doi.org/10.1038/ng.2742.

14. Dudbridge F. Power and predictive accuracy of polygenic risk scores. PLoS Genet. 2013;9(3):e1003348. doi:10.1371/journal. pgen. 1003348.

15. Cross-Disorder Group of the Psychiatric Genomics Consortium, Smoller JW, Craddock N, Kendler K, Lee PH, Neale BM, et al. Identification of risk loci with shared effects on five major psychiatric disorders: a genome-wide analysis. Lancet. 2013;381(9875): 1371-9. doi:10.1016/S0140-6736(12)62129-1.

16. Stefansson $H$, Rujescu $D$, Cichon S, Pietiläinen $\mathrm{OPH}$, Ingason $\mathrm{A}$, Steinberg S, et al. Large recurrent microdeletions associated with schizophrenia. Nature. 2008;455(7210):232-6. doi:10.1038/ nature 07229 . 
17. Szatkiewicz JP, O’Dushlaine C, Chen G, Chambert K, Moran JL, Neale BM, et al. Copy number variation in schizophrenia in Sweden. Mol Psychiatry. 2014;19(7):762-73. doi:10.1038/mp. 2014.40.

18. Pulver AE, Nestadt G, Goldberg R, Shprintzen RJ, Lamacz M, Wolyniec PS, et al. Psychotic illness in patients diagnosed with velo-cardio-facial syndrome and their relatives. J Nerv Ment Dis. 1994;182(8):476-8.

19. Levinson DF, Duan J, Oh S, Wang K, Sanders AR, Shi J, et al. Copy number variants in schizophrenia: confirmation of five previous findings and new evidence for 3q29 microdeletions and VIPR2 duplications. Am J Psychiatry. 2011;168(3):302-16. doi: 10.1176/appi.ajp.2010.10060876.

20. Purcell SM, Moran JL, Fromer M, Ruderfer D, Solovieff N, Roussos $\mathrm{P}$, et al. A polygenic burden of rare disruptive mutations in schizophrenia. Nature. 2014;506(7487):185-90. doi:10.1038/ nature12975.

21. Girard SL, Gauthier J, Noreau A, Xiong L, Zhou S, Jouan L, et al. Increased exonic de novo mutation rate in individuals with schizophrenia. Nat Genet. 2011;43(9):860-3. doi:10.1038/ng.886.

22. Xu B, Ionita-Laza I, Roos JL, Boone B, Woodrick S, Sun Y, et al. De novo gene mutations highlight patterns of genetic and neural complexity in schizophrenia. Nat Genet. 2012;44(12):1365-9. doi: 10.1038/ng.2446.

23. Gulsuner S, Walsh T, Watts AC, Lee MK, Thornton AM, Casadei $\mathrm{S}$, et al. Spatial and temporal mapping of de novo mutations in schizophrenia to a fetal prefrontal cortical network. Cell. 2013;154(3):518-29. doi:10.1016/j.cell.2013.06.049.

24. Fromer M, Pocklington AJ, Kavanagh DH, Williams HJ, Dwyer S, Gormley P, et al. De novo mutations in schizophrenia implicate synaptic networks. Nature. 2014;506(7487):179-84. doi:10.1038/ nature12929.

25. Bosch JA, Rector JL, Turner JE, Riddell NE, o'Hartaigh B, Burns VE. In: Psychoneuromicrobiology: Cytomegalovirus Infection as a Putative Link Between Stress, Aging, and Immunity. Springer; 2013. p. 81-100.

26. Demmler GJ. Infectious Diseases Society of America and Centers for Disease Control. Summary of a workshop on surveillance for congenital cytomegalovirus disease. Rev Infect Dis. 1991;13(2): 315-29.

27. Slavuljica I, Kvestak D, Huszthy PC, Kosmac K, Britt WJ, Jonjic S. Immunobiology of congenital cytomegalovirus infection of the central nervous system - the murine cytomegalovirus model. Cellular \& molecular immunology. 2014;.

28. Schoenfisch AL, Dollard SC, Amin M, Gardner LI, Klein RS, Mayer K, et al. Cytomegalovirus (CMV) shedding is highly correlated with markers of immunosuppression in CMV-seropositive women. J Med Microbiol. 2011;60(6):768-74.

29. Nelson CT, Demmler GJ. Cytomegalovirus infection in the pregnant mother, fetus, and newborn infant. Clin Perinatol. 1997;24(1): $151-60$.

30. Damato EG, Winnen CW. Cytomegalovirus infection: perinatal implications. J Obstet Gynecol Neonatal Nurs. 2002;31(1):86-92. doi:10.1111/j.1552-6909.2002.tb00026.x.

31. Houenou J, d'Albis MA, Daban C, Hamdani N, Delavest M, Lépine JP, et al. Cytomegalovirus seropositivity and serointensity are associated with hippocampal volume and verbal memory in schizophrenia and bipolar disorder. Prog Neuro-Psychopharmacol Biol Psychiatry. 2014;48:142-8.

32. Dollard SC, Staras SA, Amin MM, Schmid DS, Cannon MJ. National prevalence estimates for cytomegalovirus IgM and IgG avidity and association between high $\operatorname{IgM}$ antibody titer and low IgG avidity. Clin Vaccine Immunol. 2011;18(11):1895-9.

33. Deakin J, Lennox BR, Zandi MS. Antibodies to the $N$-Methyl-DAspartate Receptor and Other Synaptic Proteins in Psychosis. Biol Psychiatry. 2014;75(4):284-91.
34. Ganguli R, Brar JS, Rabin BS. Immune abnormalities in schizophrenia: evidence for the autoimmune hypothesis. Harvard Review of Psychiatry. 1994;2(2):70-83.

35. Heath RG, Krupp IM. Schizophrenia as an immunologic disorder: I. Demonstration of antibrain globulins by fluorescent antibody techniques. Arch Gen Psychiatr. 1967;16(1):1-9.

36. Cardno AG, Marshall EJ, Coid B, Macdonald AM, Ribchester TR, Davies NJ, et al. Heritability estimates for psychotic disorders: the Maudsley twin psychosis series. Arch Gen Psychiatry. 1999;56(2): $162-8$.

37. Torrey EF. Are we overestimating the genetic contribution to schizophrenia? Schizophr Bull. 1992;18(2):159-70.

38.• Børglum AD, Demontis D, Grove J, Pallesen J, Hollegaard M, Pedersen CB, et al. Genome-wide study of association and interaction with maternal cytomegalovirus infection identifies new schizophrenia loci. Molecular Psychiatry. 2013;Available from: http:/www.nature.com/mp/journal/vaop/ncurrent/full/ mp20132a.html. The first and so far only published example of experiment-wide significant interaction with a marker of infection in a GWAS of SCZ

39. Shirts BH, Prasad KM, Pogue-Geile MF, Dickerson F, Yolken RH, Nimgaonkar VL. Antibodies to cytomegalovirus and Herpes Simplex Virus 1 associated with cognitive function in schizophrenia. Schizophr Res. 2008;106(2-3):268-74. doi:10.1016/j.schres. 2008.07.017.

40. Kannan G, Sawa A, Pletnikov MV. Mouse models of geneenvironment interactions in schizophrenia. Neurobiol Dis. 2013;57:5-11. doi:10.1016/j.nbd.2013.05.012.

41. Fodil-Cornu N, Lee SH, Belanger S, Makrigiannis AP, Biron CA, Buller RM, et al. Ly49h-deficient C57BL/6 mice: a new mouse cytomegalovirus-susceptible model remains resistant to unrelated pathogens controlled by the NK gene complex. J Immunol. 2008;181(9):6394-405.

42. O'Kusky JR, Boyes BE, Walker DG, McGeer EG. Cytomegalovirus infection of the developing brain alters catecholamine and indoleamine metabolism. Brain Res. 1991;559(2):32230.

43. Silverman MN, Macdougall MG, Hu F, Pace TW, Raison CL, Miller AH. Endogenous glucocorticoids protect against TNFalpha-induced increases in anxiety-like behavior in virally infected mice. Mol Psychiatry. 2007;12(4):408-17.

44. McCarthy SE, Makarov V, Kirov G, Addington AM, McClellan J, Yoon S, et al. Microduplications of 16p11. 2 are associated with schizophrenia. Nat Genet. 2009;41(11):1223-7.

45. Steinberg S, de Jong S, Mattheisen M, Costas J, Demontis D, Jamain S, et al. Common variant at 16p11. 2 conferring risk of psychosis. Mol Psychiatry. 2012;19(1):108-14.

46. Melnick M, Abichaker G, Htet K, Sedghizadeh P, Jaskoll T. Small molecule inhibitors of the host cell COX/AREG/EGFR/ERK pathway attenuate cytomegalovirus-induced pathogenesis. Exp Mol Pathol. 2011;91(1):400-10.

47. Frydecka D, Karpinski P, Misiak B. Unravelling immune alterations in schizophrenia: can DNA methylation provide clues? Epigenomics. 2014;6(3):245-7.

48. Aberg KA, McClay JL, Nerella S, Clark S, Kumar G, Chen W, et al. Methylome-wide association study of schizophrenia: identifying blood biomarker signatures of environmental insults. JAMA Psychiatry. 2014;71(3):255-64. doi:10.1001/jamapsychiatry.2013. 3730.

49. Shi L, Fatemi SH, Sidwell RW, Patterson PH. Maternal influenza infection causes marked behavioral and pharmacological changes in the offspring. J Neurosci. 2003;23(1):297-302.

50. Short SJ, Lubach GR, Karasin AI, Olsen CW, Styner M, Knickmeyer RC, et al. Maternal influenza infection during pregnancy impacts postnatal brain development in the rhesus monkey. Biol Psychiatry. 2010;67(10):965-73. 
51. Nørgaard-Pedersen B, Hougaard DM. Storage policies and use of the Danish newborn screening biobank. J Inherit Metab Dis. 2007;30(4):530-6. doi:10.1007/s10545-007-0631-x.

52. Murcray CE, Lewinger JP, Gauderman WJ. Gene-environment interaction in genome-wide association studies. Am J Epidemiol. 2009;169(2):219-26. doi:10.1093/aje/kwn353.

53. Smith JD, Meehan MH, Crean J, McCann A. Alpha T-catenin (CTNNA3): a gene in the hand is worth two in the nest. Cell Mol Life Sci. 2011;68(15):2493-8.

54. Van Dijk M, Mulders J, Könst A, Janssens B, Van Roy F, Blankenstein $\mathrm{M}$, et al. Differential downregulation of $\alpha \mathrm{T}$-catenin expression in placenta: trophoblast cell type-dependent imprinting of the CTNNA3 gene. Gene Expr Patterns. 2004;5(1):61-5.

55. Pereira L, Petitt M, Tabata T. Cytomegalovirus infection and antibody protection of the developing placenta. Clin Infect Dis. 2013;57 suppl 4:S174-7.

56. Tyberghein K, Goossens S, Haigh J, Van Roy F, van Hengel J. Tissue-wide overexpression of alpha-T-catenin results in aberrant trophoblast invasion but does not cause embryonic mortality in mice. Placenta. 2012;33(7):554-60.

57. Nelissen EC, van Montfoort AP, Dumoulin JC, Evers JL. Epigenetics and the placenta. Hum Reprod Update. 2011;17(3):397-417.

58. Cannon TD, Rosso IM, Hollister JM, Bearden CE, Sanchez LE, Hadley T. A prospective cohort study of genetic and perinatal influences in the etiology of schizophrenia. Schizophr Bull. 2000;26(2):351-66.

59. Bacchelli E, Ceroni F, Pinto D, Lomartire S, Giannandrea M, D'Adamo P, et al. A CTNNA3 compound heterozygous deletion implicates a role for $\alpha \mathrm{T}$-catenin in susceptibility to autism spectrum disorder. J Neurodev Disord. 2014;6(1):17.

60. Busby V, Goossens S, Nowotny P, Hamilton G, Smemo S, Harold $\mathrm{D}$, et al. $\alpha$-T-catenin is expressed in human brain and interacts with the Wnt signaling pathway but is not responsible for linkage to chromosome 10 in Alzheimer's disease. Neruomol Med. 2004;5(2): 133-46.

61. Angelova M, Zwezdaryk K, Ferris M, Shan B, Morris CA, Sullivan DE. Human cytomegalovirus infection dysregulates the canonical Wnt/ $\beta$-catenin signaling pathway. PLoS Pathog. 2012;8(10): e1002959.

62. Razakandrainibe R, Combes V, Grau GE, Jambou R. Crossing the wall: the opening of endothelial cell junctions during infectious diseases. Int J Biochem Cell Biol. 2013;45(7):1165-73. doi:10. 1016/j.biocel.2013.03.010.

63. Betancur C, Sakurai T, Buxbaum JD. The emerging role of synaptic cell-adhesion pathways in the pathogenesis of autism spectrum disorders. Trends Neurosci. 2009;32(7):402-12. doi:10.1016/j. tins.2009.04.003.

64. Carter CJ. Interactions between the products of the Herpes simplex genome and Alzheimer's disease susceptibility genes: relevance to pathological-signalling cascades. Neurochem Int. 2008;52(6):920 34. doi:10.1016/j.neuint.2007.11.003.

65. Ertekin-Taner N, Graff-Radford N, Younkin LH, Eckman C, Baker $\mathrm{M}$, Adamson J, et al. Linkage of plasma Abeta42 to a quantitative locus on chromosome 10 in late-onset Alzheimer's disease pedigrees. Science. 2000;290(5500):2303-4. doi:10.1126/science.290. 5500.2303.

66. Myers A, Holmans P, Marshall H, Kwon J, Meyer D, Ramic D, et al. Susceptibility locus for Alzheimer's disease on chromosome 10. Science. 2000;290(5500):2304-5. doi:10.1126/science.290. 5500.2304 .

67. Kim S, Cho B, Park C, Shin E, Cho E, Yang E, et al. Alpha-Tcatenin (CTNNA3) gene was identified as a risk variant for toluene diisocyanate-induced asthma by genome-wide association analysis. Clin Exp Allergy. 2009;39(2):203-12. 